\title{
SEEDLING GROWTH OF WHEAT AS AFFECTED BY SOIL SALINITY
}

\author{
E. Sultana ${ }^{1}$, M. A. Hasan ${ }^{2}$, S. Sikder ${ }^{2}$, M. S. Rana ${ }^{2 *}$ and F. Alam ${ }^{3}$ \\ ${ }^{1}$ Chandgong Degree College, Dinajpur \\ ${ }^{2}$ Department of Crop Physiology and Ecology, Hajee Mohammad Danesh Sci. and Tech. University, Dinajpur \\ ${ }^{3}$ Office of the Registrar, Hajee Mohammad Danesh Science and Technology University, Dinajpur \\ *Corresponding author, E-mail: sohel0901084@gmail.com
}

(Received: 29 May 2017, Accepted: 23 August 2017)

Keywords: Wheat genotypes, artificially developed soil salinity and seedling growth

\begin{abstract}
An experiment was conducted to evaluate seedling growth of wheat under saline condition. Thirty wheat genotypes were grown in trays containing different levels of salinity (Control, 6 and $12 \mathrm{dSm}^{-1}$ ) during October to December, 2013. Seedling emergence index, shoot and root length, shoot and root dry weight were found to be reduced with the increases of soil salinity level but the degree of reduction were not similar for all wheat genotypes. Salt tolerance index (STI) also indicated a wide difference in salt tolerance among the wheat genotypes. Sourav, Gourav, Shatabdi, BAW 1185, BAW 1186, BAW 1187, BAW 1189 and BAW 1193 were more salt tolerance while BAW 1177, BAW 1190 and BAW 1198 showed greater salt sensitivity than the other wheat genotypes at $6 \mathrm{dS} \mathrm{m}^{-1}$. However, at $12 \mathrm{dS} \mathrm{m}^{-1}$, Sourav, Gourav, Shatabdi, Sufi and BAW 1184 showed more salt tolerance and BAW 1183, BAW 1190, BAW 1192, BAW 1194 and BAW 97 provided greater stress sensitivity among the testing wheat genotypes. Considering both saline stress Sourav, Gourav and Shatabdi were found to be salt tolerant and BAW 1190 was saline sensitive wheat genotypes.
\end{abstract}

\section{Introduction}

Salt stress affects seedling growth in different ways depending on plant species (Gul and Weber 1999). Salinity affects the seedling growth of plants (Tezara et al., 2003, Rahman and Kayani, 1988 ) by slow or less mobilization of reserve foods (Kayani et al., 1990) suspending the cell division, enlargement (Meiri and Poljakoff - Mayber, 1970) and injuring hypocotyls (Assadian and Miyamoto, 1987). Generally, Salinity can inhibit plant growth by three major ways (Greenway and Munns, 1980): a) Water deficit arising from the more negative water potential (elevated osmotic pressure) of the soil solution; b) Specific ion toxicity usually associated with either excessive chloride or sodium uptake; and c) Nutrient ion imbalance when the excess of $\mathrm{Na}+$ or $\mathrm{Cl}$ - leads to a diminished uptake of $\mathrm{K}^{+}, \mathrm{Ca}^{2+}, \mathrm{NO}_{3}{ }^{-}$or $\mathrm{P}$, or to impaired internal distribution of one or another of these ions. Therefore, Boubaker (1996) showed that seedling characteristics are also viable criteria for selecting salt tolerance in wheat in a screening experiment of wheat cultivars. The relation of various seedling growth parameters to seed yield and yield component under saline conditions are important for the development of salt tolerant cultivar. The screening of salt tolerant lines/cultivars has been attempted by many researchers on various species at seedling growth stage (Ashraf, 1999). Therefore, the present study was conducted to evaluate the response of wheat genotypes to artificially developed saline soil at seedling stage. 
Sultana et al.

\section{Materials and Methods}

The experiment was conducted on tray at the research field of the department of Crop Physiology and Ecology, Hajee Mohammad Danesh Science and Technology University (HSTU), Dinajpur during October 2013 to December 2013. The top soil (0-15 cm depth) was collected from research field of HSTU, Dinajpur. The soil was sandy loam and Cation Exchange Capacity (CEC) and $\mathrm{pH}$ value of the soil were $5.60 \mathrm{meq} / 100 \mathrm{~g}$ and 5.45 respectively. The soil contained $1.19 \%$ organic matter, $0.69 \%$ organic carbon, $0.07 \%$ total Nitrogen, 16.75 ppm available Phosphorous and 0.17 me $/ 100 \mathrm{~g}$ exchangeable Potassium (The soil was analysed at SRDI, Dinajpur). Each of 21 plastic bowl (diameter $18 \mathrm{~cm}$ ) was filled with $10 \mathrm{~kg}$ of air dried soil. Different amount $(0,0.2,0.4,0.6,0.8,1.0$, and $1.2 \% \mathrm{~W} / \mathrm{W})$ of $\mathrm{NaCl}$ and $\mathrm{Na}_{2} \mathrm{SO}_{4}$ in a ratio of $1: 1$ was added in different plastic bowl. Each treatment was replicated thrice. Before adding, the calculated amount of salt was dissolved in 1 litre of tap water. The plastic bowl were then kept under shade for air drying and pulverized every day. After complete air drying the soil in each bowl was mixed properly. The soil samples were collected from each plastic bowl for determining the soil salinity level (Rhoades et al., 1999). The soil salinity level was plotted against the salt concentration and a standard curve was prepared which is shown in Fig.1. Artificial saline soil of different $\mathrm{dSm}^{-1}\left(0,6\right.$ and $\left.12 \mathrm{dS} \mathrm{m}^{-1}\right)$ was developed by adding calculated amount of $\mathrm{NaCl}$ and $\mathrm{Na}_{2} \mathrm{SO}_{4}$ in a ratio of $1: 1$ with the help of standard curve (Fig.1).

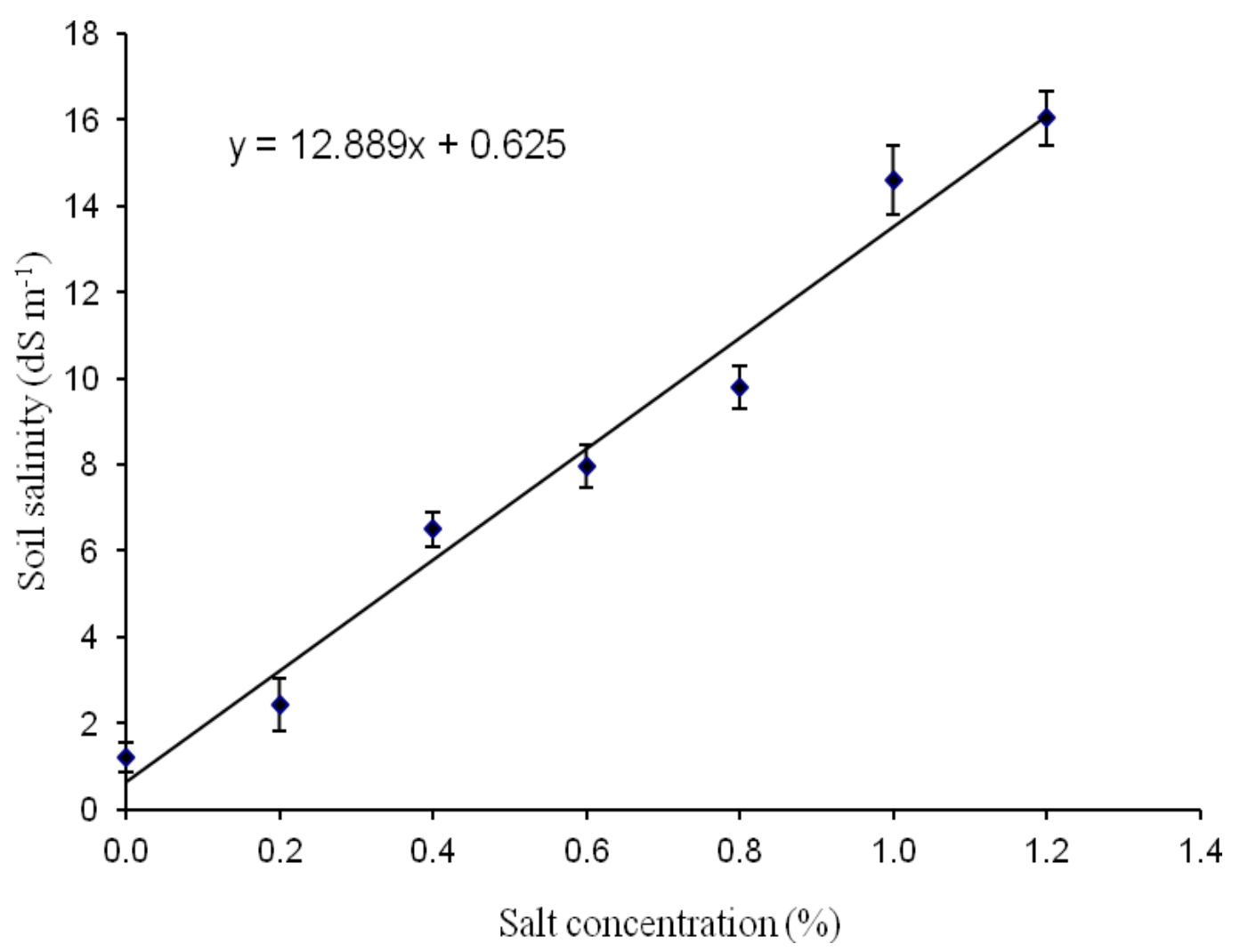

Fig.1. Relationship between soil salinity and salt concentration added in the soil. 
Eighteen try of $1.5 \mathrm{~m} \times 0.6 \mathrm{~m}$ size was filled with artificially developed saline soil of different $\mathrm{dS}$ $\mathrm{m}^{-1}\left(0,6\right.$ and $\left.12 \mathrm{dS} \mathrm{m}^{-1}\right)$. For the placement of seeds of thirty wheat genotypes in different

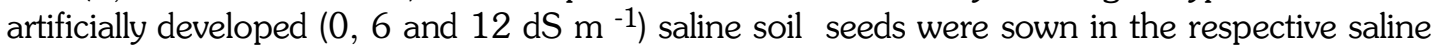
soil containing trays at the depth of $3 \mathrm{~cm}$. Fifty seeds were sown in each line and there were 15 lines in each tray and every line contained single genotype. For thirty wheat genotypes two trays were required to complete a single treatment the experiment was replicated thrice having eighteen trays. Growing of seedlings were allowed up to 30 days under an artificial shade or net house.

Intercultural operation like weeding was done to maintain normal growth of the crop. Normal irrigation was given in each tray in every alternate day until harvesting. At 30 days after sowing, five seedlings from each treatment combinations were sampled. Shoot and root length of individual seedling were recorded manually with scale. Then the seedlings were dried separately at $70 \mathrm{C}$ for $72 \mathrm{~h}$ in an electric oven (Model- 03-54639, Binder, Germany) and weight were recorded with an electrical balance (Model- AND EK-300 i).

Salt Tolerance Index was calculated using following formula as Goudarzi and Pakniyat (2008)

$$
\mathrm{STI}=\frac{\text { Performance under salt stress condition }}{\text { Performance under normal stress condition }}
$$

The data were analyzed by partitioning the total variance with the help of computer using MSTATC program. The treatment means were compared using Tukey's Test.

\section{Results and Discussion}

\section{Emergence index}

Emergence index which indicates the speed of emergence was significantly influenced by interaction effect of salinity levels and wheat genotypes (Table 1). Emergence index was higher at control (with a range from 54.61 in Gourav to 71.44 in BAW-1182 and a mean of 63.70) moderate at moderate stress (with a range from 40.78 in BAW 1199 to 62.11 in BAW 1182 and a mean of 49.43) and lower at higher saline stress (with a range from 4.46 in BAW 1199 to 29.16 in BARI Gom 25 and a mean of 19.03). The results showed that the speed of emergence was reduced with the increment of soil salinity stress but the degree of reduction in emergence index was not similar for all wheat genotypes at moderate and higher salinity stress compared to control. At moderate soil salinity stress, wheat genotypes- Sourav,Sufi, Bijoy, Prodip, BARI Gom 25, BARI Gom 26, BARI Gom 27, BARI Gom 28, BAW 1184, BAW 1185, BAW 1187, BAW 1189, BAW 1190, BAW 1191, BAW 1192, BAW 1194, BAW 1196, BAW 1197, BAW 1198, BAW 1199, BAW 1200 and BAW 1201 showed more than $20 \%$ reduction and wheat genotypes- Gourov, BAW 1177, BAW 1182, BAW 1183, BAW 1186, BAW 1193 and BAW 1195 showed less than 20\% reduction in emergence index compared to control except in Shatabdi in which emergence index was even increased. At higher soil salinity stress, only wheat genotypes Shatabdi showed less than $50 \%$ reduction and all others genotypes showed more than $50 \%$ reduction in emergence index compared to control. 
Sultana et al.

Table 1. Emergence index of different wheat genotypes as influenced by salinity levels

\begin{tabular}{|c|c|c|c|c|c|}
\hline \multirow[b]{2}{*}{$\begin{array}{l}\text { Wheat } \\
\text { genotypes }\end{array}$} & \multicolumn{3}{|c|}{ Emergence index } & \multirow{2}{*}{$\begin{array}{l}\text { \% change } \\
\text { over control } \\
\text { at } 6 \mathrm{dS} \mathrm{m}^{-1}\end{array}$} & \multirow{2}{*}{$\begin{array}{c}\text { \% change } \\
\text { over } \\
\text { control at } \\
12 \mathrm{dS} \mathrm{m}^{-1}\end{array}$} \\
\hline & Control & $\begin{array}{c}\text { Saline } \\
\left(6 \mathrm{dS} \mathrm{m}^{-1}\right)\end{array}$ & $\begin{array}{c}\text { Saline } \\
\left(12 \mathrm{dS} \mathrm{m}^{-1}\right)\end{array}$ & & \\
\hline Sourav & 63.87 & 50.79 & 10.75 & -20.47 & -83.17 \\
\hline Gourav & 54.61 & 50.79 & 18.50 & -6.99 & -66.12 \\
\hline Shatabdi & 56.65 & 59.58 & 28.84 & +5.17 & -49.09 \\
\hline Sufi & 66.60 & 49.69 & 28.91 & -25.39 & -56.59 \\
\hline Bijoy & 67.63 & 46.54 & 22.19 & -31.18 & -67.19 \\
\hline Prodip & 64.43 & 48.81 & 14.03 & -24.24 & -78.22 \\
\hline BARI Gom-25 & 66.43 & 51.23 & 29.16 & -22.88 & -56.10 \\
\hline BARI Gom-26 & 65.22 & 46.70 & 27.90 & -28.39 & -57.22 \\
\hline BARI Gom-27 & 63.87 & 50.41 & 23.14 & -21.07 & -63.77 \\
\hline BARI Gom-28 & 69.11 & 53.77 & 16.34 & -22.19 & -76.36 \\
\hline BAW 1177 & 65.79 & 53.71 & 22.09 & -18.36 & -66.42 \\
\hline BAW 1182 & 71.44 & 62.11 & 27.82 & -13.05 & -61.06 \\
\hline BAW 1183 & 66.12 & 58.19 & 29.56 & -11.99 & -55.29 \\
\hline BAW 1184 & 69.11 & 51.61 & 24.08 & -25.32 & -65.16 \\
\hline BAW 1185 & 64.79 & 45.27 & 6.38 & -30.12 & -90.15 \\
\hline BAW 1186 & 61.21 & 58.77 & 28.80 & -3.98 & -52.95 \\
\hline BAW 1187 & 60.37 & 45.63 & 15.90 & -24.41 & -73.66 \\
\hline BAW 1189 & 60.55 & 42.28 & 9.20 & -30.17 & -84.81 \\
\hline BAW 1190 & 63.64 & 42.48 & 10.71 & -33.25 & -83.17 \\
\hline BAW 1191 & 66.34 & 46.94 & 28.47 & -29.24 & -57.08 \\
\hline BAW 1192 & 62.06 & 46.91 & 6.89 & -24.41 & -88.90 \\
\hline BAW 1193 & 58.11 & 50.81 & 24.55 & -12.56 & -57.75 \\
\hline BAW 1194 & 60.38 & 46.68 & 11.05 & -22.69 & -81.69 \\
\hline BAW 1195 & 64.06 & 51.87 & 14.11 & -19.03 & -77.97 \\
\hline BAW 1196 & 66.77 & 50.51 & 16.61 & -24.35 & -75.12 \\
\hline BAW 1197 & 67.24 & 46.59 & 17.29 & -30.71 & -74.29 \\
\hline BAW 1198 & 59.34 & 45.56 & 6.26 & -23.22 & -89.45 \\
\hline BAW 1199 & 56.16 & 40.78 & 4.46 & -27.39 & -92.06 \\
\hline BAW 1200 & 62.24 & 46.98 & 28.94 & -24.52 & -53.50 \\
\hline BAW 1201 & 66.94 & 36.90 & 18.23 & -44.88 & -72.77 \\
\hline Range & $54.61-71.44$ & $40.78-62.11$ & $4.46-29.16$ & & \\
\hline Mean & 63.70 & 49.43 & 19.03 & & \\
\hline $\operatorname{LSD}_{(0.01)}$ & & 6.25 & & & \\
\hline CV (\%) & & 8.81 & & & \\
\hline
\end{tabular}

\section{Shoot length}

Shoot length of 30 days old seedling was significantly influenced by influenced by the interaction effect of soil salinity levels and wheat genotypes (Table 2). The shoot length was found to be higher at control (with a range from $33.30 \mathrm{~cm}$ in BAW 1193 to $53.00 \mathrm{~cm}$ in BAW 1182 and a mean of $41.54 \mathrm{~cm}$ ), moderate at moderate stress (ranging from $24.20 \mathrm{~cm}$ in BARI Gom 25 to $38.97 \mathrm{~cm}$ Sourav with a mean of $29.92 \mathrm{~cm}$ ) and lower at higher soil salinity stress (with a range from $17.50 \mathrm{~cm}$ in BAW 1197 to $38.90 \mathrm{~cm}$ in Sourav and a mean of $23.63 \mathrm{~cm}$ ). The shoot length was found to be reduced with the increment of soil salinity stress but the degree of reduction was not similar for all wheat genotypes. 
Influence of Soil Salinity on Seedling Growth of Wheat

Table 2. Shoot length of different wheat genotypes as influenced by salinity levels

\begin{tabular}{|c|c|c|c|c|c|}
\hline \multirow{2}{*}{$\begin{array}{c}\text { Wheat } \\
\text { genotypes }\end{array}$} & \multicolumn{3}{|c|}{ Shoot length $(\mathrm{cm})$} & \multirow{2}{*}{$\begin{array}{c}\text { \% change over } \\
\text { control at } 6 \mathrm{dS} \\
\mathrm{m}^{-1}\end{array}$} & \multirow{2}{*}{$\begin{array}{c}\text { \% change over } \\
\text { control at } 12 \mathrm{dS} \\
\mathrm{m}^{-1}\end{array}$} \\
\hline & Control & $\begin{array}{c}\text { Saline } \\
\left(6 \mathrm{dS} \mathrm{m}^{-1}\right)\end{array}$ & $\begin{array}{c}\text { Saline } \\
\left(12 \mathrm{dS} \mathrm{m}^{-1}\right)\end{array}$ & & \\
\hline Sourav & 40.50 & 38.97 & 38.90 & -3.77 & -3.95 \\
\hline Gourav & 38.00 & 34.03 & 30.80 & -10.44 & -18.94 \\
\hline Shatabdi & 35.40 & 32.13 & 26.80 & -9.24 & -24.29 \\
\hline Sufi & 36.60 & 28.70 & 24.50 & -21.58 & -33.06 \\
\hline Bijoy & 36.60 & 25.40 & 22.00 & -30.60 & -39.89 \\
\hline Prodip & 36.80 & 24.60 & 21.00 & -33.15 & -42.93 \\
\hline BARI Gom-25 & 40.17 & 24.20 & 21.90 & -39.75 & -45.48 \\
\hline BARI Gom-26 & 39.07 & 26.20 & 21.70 & -32.94 & -44.45 \\
\hline BARI Gom-27 & 40.50 & 27.40 & 22.10 & -32.34 & -45.43 \\
\hline BARI Gom-28 & 45.50 & 29.00 & 22.20 & -36.26 & -51.20 \\
\hline BAW 1177 & 44.50 & 31.40 & 27.40 & -29.43 & -38.42 \\
\hline BAW 1182 & 53.00 & 35.40 & 24.60 & -33.20 & -53.58 \\
\hline BAW 1183 & 42.90 & 34.20 & 22.90 & -20.27 & -46.62 \\
\hline BAW 1184 & 41.80 & 30.30 & 28.10 & -27.51 & -32.77 \\
\hline BAW 1185 & 43.20 & 36.60 & 28.20 & -15.27 & -34.72 \\
\hline BAW 1186 & 47.37 & 38.30 & 27.80 & -19.14 & -41.31 \\
\hline BAW 1187 & 39.40 & 31.67 & 23.00 & -19.61 & -41.62 \\
\hline BAW 1189 & 44.30 & 32.90 & 20.50 & -25.73 & -53.72 \\
\hline BAW 1190 & 43.50 & 29.50 & 24.60 & -32.18 & -43.44 \\
\hline BAW 1191 & 40.50 & 26.80 & 19.60 & -33.82 & -51.60 \\
\hline BAW 1192 & 41.67 & 27.20 & 19.50 & -34.72 & -53.20 \\
\hline BAW 1193 & 33.30 & 26.13 & 18.80 & -21.53 & -43.54 \\
\hline BAW 1194 & 37.70 & 28.00 & 18.70 & -25.72 & -50.39 \\
\hline BAW 1195 & 40.50 & 30.10 & 21.10 & -25.67 & -47.90 \\
\hline BAW 1196 & 50.57 & 29.10 & 24.77 & -42.45 & -51.01 \\
\hline BAW 1197 & 42.60 & 28.80 & 17.50 & -32.39 & -58.92 \\
\hline BAW 1198 & 42.00 & 25.50 & 23.90 & -39.28 & -43.09 \\
\hline BAW 1199 & 42.10 & 27.63 & 17.70 & -34.37 & -57.95 \\
\hline BAW 1200 & 42.70 & 29.50 & 23.30 & -30.91 & -45.43 \\
\hline BAW 1201 & 43.70 & 28.10 & 25.10 & -35.70 & -42.56 \\
\hline Range & $33.30-$ & $24.60-$ & $17.50-$ & & \\
\hline & 53.00 & 38.97 & 38.90 & & \\
\hline Mean & 41.54 & 29.92 & 23.63 & & \\
\hline $\operatorname{LSD}_{(0.01)}$ & & 1.52 & & & \\
\hline $\mathrm{CV}(\%)$ & & 8.35 & & & \\
\hline
\end{tabular}

At moderate soil salinity stress, wheat genotypes-Sufi, Bijoy, Prodip, BARI Gom 25, BARI Gom 26, BARI Gom 27, BARI Gom 28, BAW 1177, BAW 1182, BAW 1183, BAW 1184, BAW 1189, BAW 1190, BAW 1191, BAW 1192, BAW 1194, BAW 1195, BAW 1196, BAW1197, BAW 1198, BAW1199, BAW 1200 and BAW1201 showed more than 20\% reduction and wheat genotypes- Sourav, Gourav, Shatabdi, BAW 1185, BAW 1186 and BAW 1187 showed less than 20\% reduction in shoot length compared to control. At higher soil salinity stress, wheat genotypes- BARI Gom 28, BAW 1182, BAW 1189, BAW 1191, BAW 1192, BAW 1194, BAW 1196, BAW 1197 and BAW 1199 showed more than 50\% reduction and others wheat genotypes showed less than 50\% reduction in shoot length compared to control. 
Sultana et al.

\section{Root length}

Root length of 30 days old seedling was significantly influenced by the interaction effect of soil salinity levels and wheat genotypes (Table 3). The root length was found to be higher at control (with a range from $12.57 \mathrm{~cm}$ in Sufi to $23.20 \mathrm{~cm}$ in BAW 1185 and a mean of $17.03 \mathrm{~cm}$ ), moderate at moderate stress (ranging from $8.03 \mathrm{~cm}$ in BAW 1194 to $16.23 \mathrm{~cm}$ in Gourov with a mean of $12.99 \mathrm{~cm}$ ) and lower at higher soil salinity stress (with a range from $6.33 \mathrm{~cm}$ in BAW 1200 to $13.70 \mathrm{~cm}$ in Shatabdi and a mean of $9.44 \mathrm{~cm}$ ).

Table 3. Root length of different wheat genotypes as influenced by salinity levels

\begin{tabular}{|c|c|c|c|c|c|}
\hline \multirow[b]{2}{*}{ Wheat genotypes } & \multicolumn{3}{|c|}{ Root length $(\mathrm{cm})$} & \multirow{2}{*}{$\begin{array}{c}\text { \% change over } \\
\text { control at } 6 \mathrm{dS} \\
\mathrm{m}^{-1}\end{array}$} & \multirow{2}{*}{$\begin{array}{c}\% \text { change } \\
\text { over control at } \\
12 \mathrm{dS} \mathrm{m}^{-1}\end{array}$} \\
\hline & Control & $\begin{array}{l}\text { Saline } \\
\left(6 \mathrm{dS} \mathrm{m}^{-1}\right)\end{array}$ & $\begin{array}{c}\text { Saline } \\
\left(12 \mathrm{dS} \mathrm{m}^{-1}\right)\end{array}$ & & \\
\hline Sourav & 14.13 & 13.70 & 11.50 & -3.04 & -18.61 \\
\hline Gourav & 14.00 & 16.23 & 11.20 & +15.93 & -20.00 \\
\hline Shatabdi & 12.84 & 13.20 & 13.70 & +2.80 & +6.70 \\
\hline Sufi & 12.57 & 12.70 & 8.33 & +1.03 & -33.73 \\
\hline Bijoy & 18.00 & 14.60 & 8.43 & -18.89 & -53.17 \\
\hline Prodip & 18.00 & 14.50 & 12.20 & -19.44 & -32.22 \\
\hline BARI Gom-25 & 17.80 & 12.60 & 11.28 & -29.21 & -36.62 \\
\hline BARI Gom-26 & 16.10 & 13.00 & 11.60 & -19.25 & -27.95 \\
\hline BARI Gom-27 & 17.30 & 13.80 & 9.60 & -20.23 & -44.51 \\
\hline BARI Gom-28 & 16.30 & 12.70 & 11.50 & -22.09 & -29.45 \\
\hline BAW 1177 & 19.00 & 14.00 & 12.00 & -26.32 & -36.84 \\
\hline BAW 1182 & 14.80 & 12.80 & 9.70 & -13.51 & -34.46 \\
\hline BAW 1183 & 19.60 & 14.50 & 11.10 & -26.02 & -43.37 \\
\hline BAW 1184 & 18.00 & 10.40 & 11.70 & -42.22 & -35.00 \\
\hline BAW 1185 & 23.20 & 16.10 & 12.00 & -30.60 & -48.28 \\
\hline BAW 1186 & 18.50 & 8.30 & 6.73 & -55.14 & -63.62 \\
\hline BAW 1187 & 21.30 & 11.40 & 7.83 & -46.48 & -63.24 \\
\hline BAW 1189 & 16.00 & 8.13 & 7.20 & -49.19 & -55.00 \\
\hline BAW 1190 & 18.60 & 9.83 & 8.50 & -47.15 & -54.30 \\
\hline BAW 1191 & 17.30 & 13.10 & 6.90 & -24.27 & -60.12 \\
\hline BAW 1192 & 18.03 & 13.30 & 6.53 & -26.23 & -63.78 \\
\hline BAW 1193 & 16.40 & 14.40 & 7.60 & -12.19 & -53.66 \\
\hline BAW 1194 & 16.30 & 8.03 & 7.30 & -50.74 & -55.21 \\
\hline BAW 1195 & 16.30 & 14.30 & 8.00 & -12.27 & -50.92 \\
\hline BAW 1196 & 17.30 & 11.40 & 10.20 & -34.10 & -41.04 \\
\hline BAW 1197 & 14.90 & 15.00 & 8.80 & +0.67 & -40.94 \\
\hline BAW 1198 & 17.20 & 11.50 & 9.53 & -33.14 & -44.59 \\
\hline BAW 1199 & 18.00 & 15.60 & 7.70 & -13.33 & -57.22 \\
\hline BAW 1200 & 16.00 & 14.50 & 6.33 & -9.38 & -60.44 \\
\hline BAW 1201 & 17.40 & 16.10 & 8.30 & -7.47 & -52.30 \\
\hline Range & 12.57- & & $6.33-13.70$ & & \\
\hline & 23.20 & $8.03-16.23$ & & & \\
\hline Mean & 17.03 & 12.99 & 9.44 & & \\
\hline $\operatorname{LSD}_{(0.01)}$ & & 2.68 & & & \\
\hline CV (\%) & & 12.71 & & & \\
\hline
\end{tabular}


Influence of Soil Salinity on Seedling Growth of Wheat

The root length was found to be reduced with the increment of soil salinity stress but the degree of reduction was not similar for all wheat genotypes. At moderate soil salinity stress, wheat genotypes- BARI Gom 25, BARI Gom 27, BARI Gom 28, BAW 1177, BAW 1183, BAW 1184, BAW 1185, BAW 1186, BAW 1187, BAW 1189, BAW 1190, BAW 1191, BAW 1192, BAW 1194, BAW 1196 and BAW 1198 showed more than 20\% reduction and wheat genotypes- Sourav, Bijoy, Prodip, BARI Gom 26, BAW 1182, BAW 1193, BAW 1195, BAW 1199, BAW 1200 and BAW 12001 showed less than 20\% reduction in germination index compared to control except Gourov, Sufi and BAW 1197 in which root length was even increased. At higher soil salinity stress, wheat genotypes- Bijoy, BAW 1186, BAW 1187, BAW 1189, BAW 1190, BAW 1191, BAW 1192, BAW 1193, BAW 1194, BAW 1195, BAW 1199, BAW 1200 and BAW 1201 showed more than 50\% reduction and others wheat genotypes showed less than $50 \%$ reduction in root length compared to control.

\section{Shoot dry weight}

Shoot dry weight of 30 days old seedling was significantly influenced by influenced by the interaction effect of soil salinity levels and wheat genotypes (Table 4). The shoot dry weight was found to be higher at control (with a range from $2.34 \mathrm{mg}$ in Sufi to $5.01 \mathrm{mg}$ in BAW 1200 and a mean of $3.33 \mathrm{mg}$ ), moderate at moderate stress (ranging from $0.64 \mathrm{mg}$ in BARI Gom 26 to $3.18 \mathrm{mg}$ in Sourav with a mean of $1.40 \mathrm{mg}$ ) and lower at higher soil salinity stress (with a range from $0.32 \mathrm{mg}$ in BAW 1197 to $1.31 \mathrm{mg}$ in BAW 1201 and a mean of $0.67 \mathrm{mg}$ ). Shoot dry weight was found to be reduced with the increment of soil salinity stress but the degree of reduction was not similar for all wheat genotypes.

Table 4. Shoot dry weight of different wheat genotypes as influenced by salinity levels

\begin{tabular}{lccccc}
\hline \multirow{2}{*}{$\begin{array}{c}\text { Wheat } \\
\text { genotypes }\end{array}$} & Control & $\begin{array}{c}\text { Saline } \\
\left(6 \mathrm{dSm}^{-1}\right)\end{array}$ & $\begin{array}{c}\text { Saline } \\
\left(12 \mathrm{dSm}^{-1}\right)\end{array}$ & $\begin{array}{c}\text { \% change } \\
\text { over } \\
\text { control at } \\
6 \mathrm{dSm}^{-1}\end{array}$ & $\begin{array}{c}\text { \% change } \\
\text { over control } \\
\text { at } 12 \mathrm{dSm}^{-1}\end{array}$ \\
\cline { 2 - 5 } Sourav & 2.73 & 3.18 & 1.27 & +16.48 & -53.47 \\
Gourav & 2.70 & 1.41 & 0.93 & -47.77 & -65.55 \\
Shatabdi & 2.62 & 1.45 & 1.03 & -44.65 & -60.68 \\
Sufi & 2.34 & 1.13 & 0.70 & -51.70 & -70.08 \\
Bijoy & 3.29 & 1.30 & 0.63 & -60.48 & -80.85 \\
Prodip & 3.17 & 0.88 & 0.60 & -72.24 & -81.07 \\
BARI Gom-25 & 3.23 & 0.82 & 0.48 & -74.61 & -85.13 \\
BARI Gom-26 & 2.68 & 0.64 & 0.45 & -76.11 & -83.21 \\
BARI Gom-27 & 2.73 & 0.78 & 0.38 & -71.42 & -86.08 \\
BARI Gom-28 & 2.72 & 1.01 & 0.32 & -62.86 & -88.23 \\
BAW 1177 & 3.44 & 1.46 & 0.65 & -57.55 & -81.10 \\
BAW 1182 & 3.73 & 1.29 & 0.58 & -65.42 & -84.45 \\
BAW 1183 & 4.46 & 1.45 & 0.49 & -67.48 & -89.01 \\
BAW 1184 & 2.86 & 1.23 & 1.17 & -56.99 & -59.09 \\
BAW 1185 & 2.57 & 1.41 & 1.31 & -45.14 & -49.02 \\
BAW 1186 & 3.99 & 2.84 & 1.02 & -28.82 & -74.43 \\
\hline
\end{tabular}


Sultana et al.

\begin{tabular}{|c|c|c|c|c|c|}
\hline \multirow[b]{2}{*}{$\begin{array}{c}\text { Wheat } \\
\text { genotypes }\end{array}$} & \multicolumn{3}{|c|}{ Shoot dry weight (mg) } & \multirow{2}{*}{$\begin{array}{c}\% \text { change } \\
\text { over } \\
\text { control at } \\
6 \mathrm{dSm}^{-1}\end{array}$} & \multirow{2}{*}{$\begin{array}{l}\text { \% change } \\
\text { over control } \\
\text { at } 12 \mathrm{dSm}^{-1}\end{array}$} \\
\hline & Control & $\begin{array}{c}\text { Saline } \\
\left(6 \mathrm{dSm}^{-1}\right)\end{array}$ & $\begin{array}{c}\text { Saline } \\
\left(12 \mathrm{dSm}^{-1}\right)\end{array}$ & & \\
\hline BAW 1187 & 2.86 & 1.74 & 0.76 & -39.16 & -73.42 \\
\hline BAW 1189 & 2.50 & 1.78 & 0.35 & -28.80 & -86.00 \\
\hline BAW 1190 & 3.79 & 1.03 & 0.44 & -72.82 & -88.39 \\
\hline BAW 1191 & 2.80 & 1.22 & 0.41 & -56.43 & -85.35 \\
\hline BAW 1192 & 3.37 & 1.27 & 0.36 & -62.31 & -89.31 \\
\hline BAW 1193 & 3.13 & 1.65 & 0.40 & -47.28 & -87.22 \\
\hline BAW 1194 & 3.98 & 1.36 & 0.36 & -65.83 & -90.95 \\
\hline BAW 1195 & 2.77 & 1.27 & 0.49 & -54.15 & -82.31 \\
\hline BAW 1196 & 3.32 & 1.30 & 0.82 & -60.84 & -75.30 \\
\hline BAW 1197 & 3.68 & 1.37 & 0.32 & -62.77 & -91.30 \\
\hline BAW 1198 & 3.88 & 1.40 & 0.75 & -63.92 & -80.67 \\
\hline BAW 1199 & 4.49 & 1.27 & 0.56 & -71.71 & -87.53 \\
\hline BAW 1200 & 5.01 & 1.59 & 0.91 & -68.26 & -81.83 \\
\hline BAW 1201 & 5.23 & 1.50 & 1.13 & -71.32 & -78.39 \\
\hline Range & $2.34-5.01$ & $0.64-3.18$ & $0.32-1.13$ & & \\
\hline Mean & 3.33 & 1.40 & 0.67 & & \\
\hline $\operatorname{LSD}_{(0.01)}$ & & 0.087 & & & \\
\hline CV (\%) & & 8.35 & & & \\
\hline
\end{tabular}

At moderate soil salinity stress, wheat genotypes-Sufi, Bijoy, Prodip, BARI Gom 25, BARI Gom 26, BARI Gom 27, BARI Gom 28, BAW 1177, BAW 1182, BAW 1183, BAW 1184, BAW 1190, BAW 1191, BAW 1192, BAW 1194, BAW 1195, BAW 1196, BAW1197, BAW 1198, BAW1199, BAW 1200 and BAW1201 showed more than 50\% reduction and wheat genotypes- Gourav, Shatabdi, BAW 1185, BAW 1186, BAW 1187, BAW 1189 and BAW 1193 showed less than 50\% reduction in shoot dry weight compared to control except Sourav, in which shoot dry weight was even increased.At higher soil salinity stress, wheat genotypes- Bijoy, Prodip, BARI Gom 25, BARI Gom 26, BARI Gom 27, BARI Gom 28, BAW 1177, BAW 1182, BAW 1183, BAW 1189, BAW 1190, BAW 1191, BAW 1192, BAW 1193, BAW 1194, BAW 1195, BAW 1197, BAW 1198, BAW 1199 and BAW 1200 showed more than $80 \%$ reduction and others wheat genotypes showed less than $80 \%$ reduction in shoot dry weight compared to control.

\section{Root dry weight}

Root dry weight of 30 days old seedling was significantly influenced by influenced by the interaction effect of soil salinity levels and wheat genotypes (Table 5). The Root dry weight was found to be higher at control (with a range from $0.55 \mathrm{mg}$ in BAW 1196 to $1.64 \mathrm{mg}$ in BAW 1201 and a mean of $0.95 \mathrm{mg}$ ), moderate at moderate stress (ranging from $0.24 \mathrm{mg}$ in BAW 1177 to $0.93 \mathrm{mg}$ in BAW 1185 with a mean of $0.39 \mathrm{mg}$ ) and lower at higher soil salinity stress (with a range from $0.05 \mathrm{mg}$ in BAW 1194 to $0.30 \mathrm{mg}$ in BAW 1186 and a mean of $0.20 \mathrm{mg})$. 
Influence of Soil Salinity on Seedling Growth of Wheat

Table 5. Root dry weight of different wheat genotypes as influenced by salinity levels

\begin{tabular}{|c|c|c|c|c|c|}
\hline \multirow[b]{2}{*}{$\begin{array}{l}\text { Wheat } \\
\text { genotypes }\end{array}$} & \multicolumn{3}{|c|}{ Root dry weight (mg) } & \multirow{2}{*}{$\begin{array}{c}\text { \% change } \\
\text { over } \\
\text { control at } \\
6 \mathrm{dSm}^{-1}\end{array}$} & \multirow{2}{*}{$\begin{array}{l}\text { \% change } \\
\text { over control } \\
\text { at } 12 \mathrm{dSm}^{-1}\end{array}$} \\
\hline & Control & $\begin{array}{l}\text { Saline } \\
\left(6 \mathrm{dSm}^{-1}\right)\end{array}$ & $\begin{array}{c}\text { Saline } \\
\left(12 \mathrm{dSm}^{-1}\right)\end{array}$ & & \\
\hline Sourav & 0.67 & 0.69 & 0.46 & +2.98 & -31.34 \\
\hline Gourav & 0.77 & 0.42 & 0.29 & -45.45 & -62.34 \\
\hline Shatabdi & 0.79 & 0.36 & 0.47 & -54.43 & -40.51 \\
\hline Sufi & 0.64 & 0.26 & 0.26 & -59.37 & -59.37 \\
\hline Bijoy & 1.14 & 0.35 & 0.24 & -69.29 & -78.94 \\
\hline Prodip & 0.88 & 0.26 & 0.23 & -70.45 & -73.86 \\
\hline BARI Gom-25 & 0.77 & 0.28 & 0.17 & -63.63 & -77.92 \\
\hline BARI Gom-26 & 0.82 & 0.25 & 0.27 & -69.51 & -67.07 \\
\hline BARI Gom-27 & 0.73 & 0.25 & 0.12 & -65.75 & -83.56 \\
\hline BARI Gom-28 & 0.75 & 0.26 & 0.15 & -65.33 & -80.00 \\
\hline BAW 1177 & 1.28 & 0.24 & 0.28 & -81.25 & -78.12 \\
\hline BAW 1182 & 1.08 & 0.33 & 0.09 & -69.44 & -91.66 \\
\hline BAW 1183 & 1.15 & 0.45 & 0.16 & -60.87 & -86.08 \\
\hline BAW 1184 & 1.27 & 0.40 & 0.32 & -68.50 & -74.80 \\
\hline BAW 1185 & 1.71 & 0.93 & 0.27 & -45.61 & -84.210 \\
\hline BAW 1186 & 1.59 & 0.90 & 0.30 & -43.39 & -81.13 \\
\hline BAW 1187 & 0.92 & 0.40 & 0.19 & -56.52 & -79.34 \\
\hline BAW 1189 & 0.71 & 0.52 & 0.09 & -26.76 & -87.32 \\
\hline BAW 1190 & 1.02 & 0.29 & 0.16 & -71.56 & -84.31 \\
\hline BAW 1191 & 0.94 & 0.43 & 0.12 & -54.25 & -87.23 \\
\hline BAW 1192 & 0.72 & 0.31 & 0.12 & -56.94 & -83.33 \\
\hline BAW 1193 & 0.81 & 0.47 & 0.09 & -41.97 & -88.88 \\
\hline BAW 1194 & 0.85 & 0.25 & 0.05 & -70.58 & -94.11 \\
\hline BAW 1195 & 0.72 & 0.33 & 0.12 & -54.16 & -83.33 \\
\hline BAW 1196 & 0.55 & 0.34 & 0.20 & -38.18 & -63.63 \\
\hline BAW 1197 & 0.86 & 0.33 & 0.09 & -61.62 & -89.53 \\
\hline BAW 1198 & 0.90 & 0.27 & 0.17 & -70.00 & -81.11 \\
\hline BAW 1199 & 1.09 & 0.45 & 0.12 & -58.71 & -88.99 \\
\hline BAW 1200 & 0.87 & 0.50 & 0.15 & -42.52 & -82.75 \\
\hline BAW 1201 & 1.64 & 0.45 & 0.18 & -72.56 & -89.02 \\
\hline Range & $0.55-1.64$ & $0.24-0.93$ & $0.05-0.30$ & & \\
\hline Mean & 0.95 & 0.39 & 0.20 & & \\
\hline $\operatorname{LSD}_{(0.01)}$ & & 0.025 & & & \\
\hline CV $(\%)$ & & 7.76 & & & \\
\hline
\end{tabular}

Shoot dry weight was found to be reduced with the increment of soil salinity stress but the degree of reduction was not similar for all wheat genotypes. At moderate soil salinity stress, wheat genotypes- Shatabdi, Sufi, Bijoy, Prodip, BARI Gom 25, BARI Gom 26, BARI Gom 27, 
Sultana et al.

BARI Gom 28, BAW 1177, BAW 1182, BAW 1183, BAW 1184, BAW 1187, BAW 1190, BAW 1191, BAW 1192, BAW 1194, BAW 1195, BAW1197, BAW 1198, BAW 1199 and BAW1201 showed more than 50\% reduction and wheat genotypes- Gourav, BAW 1185, BAW 1186, BAW 1187, BAW 1189, BAW 1193, BAW 1196 and BAW 1200 showed less than $50 \%$ reduction in root dry weight compared to control except Sourav, in which root dry weight was even increased. At higher soil salinity stress, wheat genotypes- BARI Gom 27, BARI Gom 28, BAW 1182, BAW 1183, BAW 1185, BAW 1186, BAW 1189, BAW 1190, BAW 1191, BAW 1192, BAW 1193, BAW 1194, BAW 1195, BAW 1197, BAW 1198, BAW 1199, BAW 1200 and BAW 1201 showed more than 80\% reduction and others wheat genotypes showed less than $80 \%$ reduction in root dry weight compared to control.

\section{Seedling dry weight}

Seedling dry weight of 30 days old seedling was significantly influenced by influenced by the interaction effect of soil salinity levels and wheat genotypes (Table 6). The seedling dry weight was found to be higher at control (with a range from $3.00 \mathrm{mg}$ in Sufi to $6.87 \mathrm{mg}$ in BAW 1201 and a mean of $4.34 \mathrm{mg}$ ), moderate at moderate stress (ranging from $0.94 \mathrm{mg}$ in BARI Gom 26 to $4.34 \mathrm{mg}$ in BAW 1185 with a mean of $1.84 \mathrm{mg}$ ) and lower at higher soil salinity stress (with a range from $0.41 \mathrm{mg}$ in BAW 1194 to $1.73 \mathrm{mg}$ in Sourav and a mean of 0.89 $\mathrm{mg}$ ). Seedling dry weight was found to be reduced with the increment of soil salinity stress but the degree of reduction was not similar for all wheat genotypes. At moderate soil salinity stress, wheat genotypes- Sufi, Bijoy, Prodip, BARI Gom 25, BARI Gom 26, BARI Gom 27, BARI Gom 28, BAW 1177, BAW 1182, BAW 1183, BAW 1184, BAW 1190, BAW 1191, BAW 1192, BAW 1194, BAW 1195, BAW 1196, BAW1197, BAW 1198, BAW 1199, BAW 1200 and BAW1201 showed more than 50\% reduction and wheat genotypes- Gourav, BAW 1185, BAW 1186, BAW 1187, BAW 1189 and BAW 1193 showed less than 50\% reduction in seedling dry weight compared to control except Sourav, in which seedling dry weight was even increased. At higher soil salinity stress, wheat genotypes- Prodip, Bijoy, BARI Gom 25, BARI Gom 26, BARI Gom 27, BARI Gom 28, BAW 1177, BAW 1182, BAW 1183, BAW 1189, BAW 1190, BAW 1191, BAW 1192, BAW 1193, BAW 1194, BAW 1195, BAW 1197, BAW 1198, BAW 1199, BAW 1200 and BAW 1201 showed more than $80 \%$ reduction and others wheat genotypes showed less than $80 \%$ reduction in seedling dry weight compared to control. At higher soil salinity stress Sourov, Shatabdi, Sufi, BAW1184, BAW1186, BAW1187 and BAW11896 showed comparatively lower reduction in seedling dry weight.

In the present study, all parameters were affected due to salt stress. With increasing salt concentration in the environment for germination, shoot and root length and dry weight were reduced significantly. Garciarrubio et al. (2003) and Datta et al. (2009) were also found the same result. It has been reported that salinity stress significantly reduced net photosynthetic rates, increased energy losses for salt exclusion mechanism, largely decreased nutrient mobilization, suspending the cell division and enlargement and finally reduced plant growth (Meiri and Poljakoff-Mayber, 1970, Long and Baker, 1986 and Seeman and Sharkey, 1986). Salinity affects the seedling growth of plants (Tezara et al., 2003, Rahman and Kayani, 1988) by slow or less mobilization of reserve foods (Kayani et al., 1990), suspending the cell division, enlargement (Meiri and APoljakoff-Mayber, 1970) and injuring hypocotyls (Assadian and Miyamoto, 1987). 
Influence of Soil Salinity on Seedling Growth of Wheat

Table 6. Seedling dry weight of different wheat genotypes as influenced by salinity levels

\begin{tabular}{|c|c|c|c|c|c|}
\hline \multirow[b]{2}{*}{ Wheat genotypes } & \multicolumn{3}{|c|}{ Seedling dry weight (mg) } & \multirow{2}{*}{$\begin{array}{c}\text { \% change } \\
\text { over } \\
\text { control at } \\
6 \mathrm{dSm}^{-1}\end{array}$} & \multirow{2}{*}{$\begin{array}{l}\text { \% change } \\
\text { over control } \\
\text { at } 12 \mathrm{dSm}^{-1}\end{array}$} \\
\hline & Control & $\begin{array}{c}\text { Saline } \\
\left(6 \mathrm{dSm}^{-1}\right)\end{array}$ & $\begin{array}{c}\text { Saline } \\
\left(12 \mathrm{dSm}^{-1}\right)\end{array}$ & & \\
\hline Sourav & 3.40 & 3.87 & 1.73 & +13.82 & -49.12 \\
\hline Gourav & 3.49 & 1.83 & 1.27 & -47.56 & -63.61 \\
\hline Shatabdi & 3.42 & 1.87 & 1.50 & -45.32 & -56.14 \\
\hline Sufi & 3.00 & 1.36 & 0.97 & -54.66 & -67.66 \\
\hline Bijoy & 4.43 & 1.66 & 0.90 & -62.52 & -79.68 \\
\hline Prodip & 4.05 & 1.15 & 0.84 & -71.60 & -79.25 \\
\hline BARI Gom-25 & 3.68 & 1.10 & 0.66 & -70.11 & -82.07 \\
\hline BARI Gom-26 & 3.50 & 0.94 & 0.72 & -73.14 & -79.43 \\
\hline BARI Gom-27 & 3.47 & 1.04 & 0.50 & -70.03 & -85.59 \\
\hline BARI Gom-28 & 3.47 & 1.28 & 0.68 & -63.11 & -80.40 \\
\hline BAW 1177 & 4.73 & 1.20 & 0.94 & -74.63 & -80.13 \\
\hline BAW 1182 & 4.83 & 1.63 & 0.68 & -66.25 & -85.92 \\
\hline BAW 1183 & 5.61 & 1.91 & 0.65 & -65.95 & -88.41 \\
\hline BAW 1184 & 4.13 & 1.63 & 1.50 & -60.53 & -63.68 \\
\hline BAW 1185 & 5.95 & 4.34 & 1.58 & -27.06 & -73.45 \\
\hline BAW 1186 & 5.50 & 3.75 & 1.32 & -31.82 & -76.00 \\
\hline BAW 1187 & 3.79 & 2.15 & 0.95 & -43.27 & -74.93 \\
\hline BAW 1189 & 3.22 & 2.31 & 0.44 & -28.26 & -86.33 \\
\hline BAW 1190 & 4.70 & 1.32 & 0.61 & -71.91 & -87.02 \\
\hline BAW 1191 & 3.83 & 1.65 & 0.54 & -56.92 & -85.90 \\
\hline BAW 1192 & 4.29 & 1.58 & 0.49 & -63.17 & -88.57 \\
\hline BAW 1193 & 3.95 & 2.12 & 0.50 & -46.33 & -87.34 \\
\hline BAW 1194 & 4.84 & 1.61 & 0.41 & -66.74 & -91.53 \\
\hline BAW 1195 & 3.50 & 1.60 & 0.62 & -54.28 & -82.28 \\
\hline BAW 1196 & 3.88 & 1.64 & 1.02 & -57.73 & -73.71 \\
\hline BAW 1197 & 4.57 & 1.71 & 0.41 & -62.58 & -91.03 \\
\hline BAW 1198 & 4.78 & 1.18 & 0.92 & -75.31 & -80.75 \\
\hline BAW 1199 & 5.53 & 1.73 & 0.68 & -68.72 & -87.70 \\
\hline BAW 1200 & 5.89 & 2.09 & 1.07 & -64.52 & -81.83 \\
\hline BAW 1201 & 6.87 & 1.95 & 1.31 & -71.62 & -80.93 \\
\hline Range & $3.00-6.87$ & $0-94-4.34$ & $0.41-1.73$ & & \\
\hline Mean & 4.34 & 1.84 & 0.89 & & \\
\hline $\operatorname{LSD}_{(0.01)}$ & & 0.025 & & & \\
\hline CV (\%) & & 2.28 & & & \\
\hline
\end{tabular}

The same authors found that the reduction in root and shoot deve-lopment may be due to toxic effects of the higher level of $\mathrm{NaCl}$ concentration as well as unbalanced nutrient uptake by the seedlings. High level of salinity may have also inhibit the root and shoot elongation due to slowing down the water uptake for overall osmotic adjustments of the plant body under high salt stress condition. Salt stress result in a considerable decrease in the fresh and dry weights of root and shoot (Chartzoulakis \& Klapaki 2000, Parida and Das, 2005). But the adverse effect on seedling dry weight was different in different wheat genotypes which indicate different 
Sultana et al.

sensitivity of wheat genotypes to salt stress. Singh et al., (2000) and Moud and Maghsoudi (2008) also found differential sensitivity of wheat genotypes based on seedling growth in their study and Karim et al (1992) suggested that seedling growth is one of the most important characters for screening salt tolerance at early stage of growth.

Salt tolerance index based on seedling dry weight grown at $6 \mathrm{dS} \mathrm{m}^{-1}$ artificial saline soil

Salt tolerance index (STI) of thirty wheat genotypes based on seedling dry weight (30 days old) at $6 \mathrm{dS} \mathrm{m} \mathrm{m}^{-1}$ artificial saline soil is presented in Fig. 2. These STI values indicated a wide difference in salt tolerance among the wheat genotypes. Wheat genotypes- Sourav, Gourav, Shatabdi, BAW 1185, BAW 1186, BAW 1187, BAW 1189 and BAW 1193 showed more than 0.5 STI and Sufi, Bijoy, Prodip, BARI Gom 25, BARI Gom 26, BARI Gom 27, BARI Gom 28, BAW 1177, BAW 1182, BAW 1183, BAW 1184, BAW 1190, BAW 1191, BAW 1192, BAW 1194, BAW 1195, BAW 1196, BAW 1197, BAW 1198, BAW 1199 and BAW 1201 provided less than 0.50 STI.

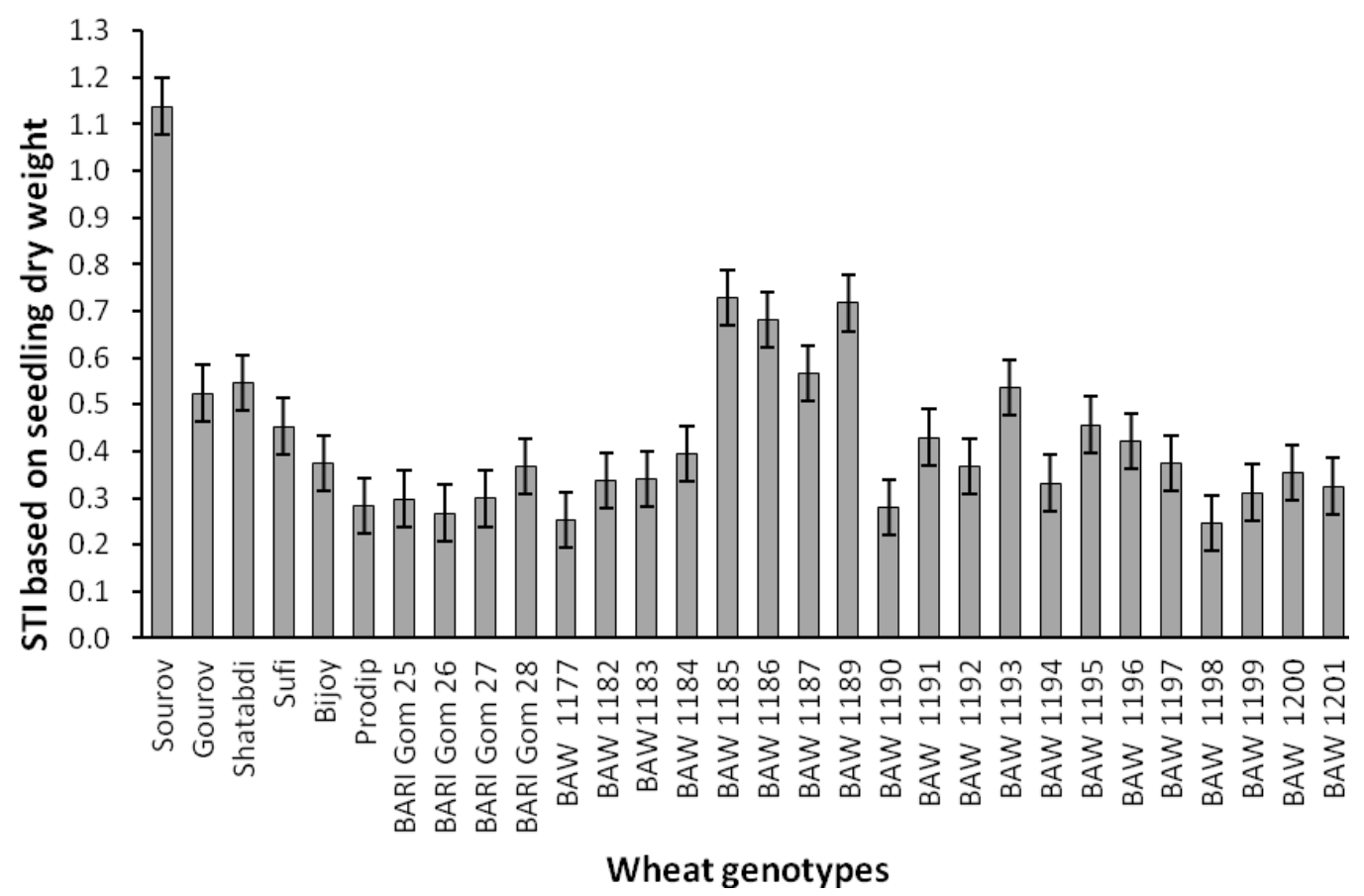

Fig. 2. Salt tolerance index of different wheat genotypes based on seedling dry weight grown at $6 \mathrm{dS}$ m-1 artificial saline soil (Vertical bar indicates LSD value).

Salt tolerance index based on seedling dry weight grown at $12 \mathrm{dS} \mathrm{m}^{-1}$ artificial saline soil

Salt tolerance index (STI) of thirty wheat genotypes based on seedling dry weight (30 days old) at $12 \mathrm{dS} \mathrm{m}^{-1}$ artificial saline soil is presented in Fig.3. These STI values indicated a wide difference in salt tolerance among the wheat genotypes. Wheat genotypes- Sourav, Gourav, Shatabdi, Sufi and BAW 1184 showed more than 0.3 STI and Bijoy, Prodip, BARI Gom 25, BARI Gom 26, BARI Gom 27, BARI Gom 28, BAW 1177, BAW 1182, BAW 1183, BAW 1185, BAW 1186, BAW 1187, BAW 1189, BAW 1190, BAW 1191, BAW 1192, BAW 
1193, BAW 1194, BAW 1195, BAW 1196, BAW 1197, BAW 1198, BAW 1199 and BAW 1201 provided less than 0.3 .

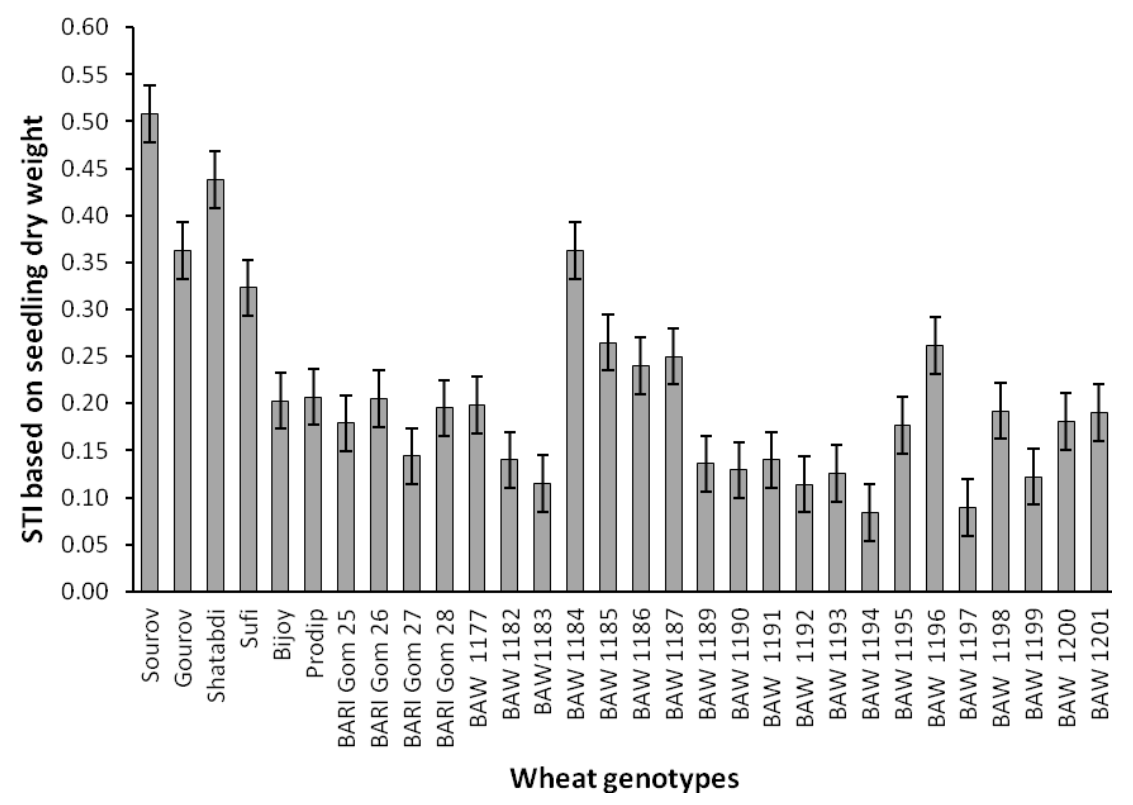

Fig.3. Salt tolerance index of different wheat genotypes based on seedling dry weight grown at $12 \mathrm{dS} \mathrm{m}^{-1}$ artificial saline soil (Vertical bar indicates LSD value).

\section{Conclusion}

Salt tolerance index (STI) also indicated a wide difference in salt tolerance among the wheat genotypes. Sourav, Gourav, Shatabdi, BAW 1185, BAW 1186, BAW 1187, BAW 1189 and BAW 1193 were more salt tolerance while BAW 1177, BAW 1190 and BAW 1198 showed greater salt sensitivity than the other wheat genotypes at $6 \mathrm{dS} \mathrm{m} \mathrm{m}^{-1}$. However, at $12 \mathrm{dS} \mathrm{m}^{-1}$, Sourav, Gourav, Shatabdi, Sufi and BAW 1184 showed more salt tolerance and BAW 1183, BAW 1190, BAW 1192, BAW 1194 and BAW 97 provided greater stress sensitivity among the testing wheat genotypes. Considering both saline stress Sourav, Gourav and Shatabdi were found to be salt tolerant and BAW 1190 was saline sensitive wheat genotypes.

\section{References}

Ashraf M. 1999. Interactive effect of salt $(\mathrm{NaCl})$ and Nitrogen form of growth, water relations and photosynthesis capacity of sunflower (Helianthus annus L.). Ann. Appl. Biol. 135: 509513.

Assadian N. W. and S. Miyamoto. 1987. Salt effects on alfalfa seedling emergence. Agron. J.79: 710-714.

Boubaker M. 1996. Salt tolerance of durum wheat cultivars during germination and early seedling growth. Agric. Medit. 126:32-39. 


\section{Sultana et al.}

Chartzoulakis K. and G. Klapaki. 2000. Response of two green house pepper hybrids to $\mathrm{NaCl}$ salinity during different growth stages. Sci. Hortic. 86: 247-260.

Datta J. K., S. Nag, A. Banerjee. and N. K. Mondal. 2009. Impact of salt stress on five varieties of Wheat (Triticum aestivum L.) cultivars under laboratory condition. J. Appl. Sci. Environ. 13(3): $93-97$.

Garciarrubio A., J. P. Leg Aria and A. A. Covarrubias. 2003. Abscisic acid inhibits germination of mature Arabidopsis seeds by limiting the availability of energy and nutrients. Planta 2:182187.

Goudarzi, M. and H. Pakniyat. 2008. Evaluation of wheat cultivars under salinity stress based on some agronomic and physiological traits. J. Agri. Soc. Sci. 4: 81-4.

Greenway H. and R. Munns. 1980. Mechanism of salt tolerance in non-halophytes. Ann. Rev. Plant Physiol. 31. 149-190.

Gul B. and D. J. Weber. 1999. Effect of salinity, light, and temperature on germination in Allenrolfea occidentalis. Can. J. Bot. 77:240- 246.

Karim M. A., N. Utsunomiya and S. Shigenaga. 1992. Effect of sodium chloride on germination and growth of hexaploid triticale at early seedling stage. Japan J. Crop Sci. 61 (2):279284.

Kayani S. A., H. H. Naqvi and I. P. Ting. 1990. Salinity effects on germination and mobilization of reserves in Jojoba seed. Crop Sci. 30 (3): 704-708.

Long S. P. and N. R. Baker. 1986. Saline Terrestrial Environments. In: N. R. Baker and S. P. Long (Eds). Photosynthesis in Contrasting Environments. Pp. 63-102. Elsevier, Amsterdam.

Meiri, A. and A. Poljakoff-Mayber. 1970. Effect of various salinity regimes on growth, leaf expansions and transpiration rate of bean plants. Plant Soil Sci. 109: 26-34.

Moud A. M. and K. Maghsoudi. 2008. Salt stress effects on respiration and growth of germinated seeds of different wheat (Triticum aestivum L.) cultivars. World J. Agril. Sci. 4(3): 351-358.

Parida A. K. and A. B. Das., 2004. Salt tolerance and salinity effect on plants: a review. Ecotoxicol. and Environ. Safely. 60: 324-349.

Rahman M. and S. A. Kayani. 1988. Effects of Chloride type of salinity on root growth and anatomy of Corn (Zea mays L.). Biologia. 34 (1): 123-131.

Rhoades J. D., F. Chanduvi and S. Lesch. 1999. Soil salinity assessment; methods and interpretation of electrical conductivity measurements. P: 5-6.

Seeman J. R. and J. D. Sharkey. 1986. Salinity and Nitrogen effects on photosynthesis, Ribulose1,5 bisphosphate carboxylase and metabolites pool size in Phaseolus vulgaris L. Plant Physiol. 82: 555-560.

Singh A. K., V. Prakash and E. V. D. Sastry. 2000. Effect of salinity stress on seed germination and seeding growth of wheat. Agric. Sci. Digest. 20(2): 96-98.

Tezara W., D. Martinez, E. Rengifo and A. Herrera. 2003. Photosynthetic response of the tropical spiny shrub Lycium nodosum (Solanaceae) to drought, soil salinity and saline spray. Annals Bot.. 92: 757-765. 